Check for updates

Cite this: RSC Adv., 2020, 10, 15228

\title{
Ru-catalysed oxidative cyclisation of 1,5-dienes: an unprecedented role for the co-oxidant $\uparrow$
}

\begin{abstract}
Aqeel A. Hussein (iD) ab
The Ru-mediated oxidative cyclisation of 1,5-dienes to furnish 2,5-dihydroxyalkyl-substituted tetrahydrofuran-diols (THF-diols) represents a practical approach for the synthesis of many bioactive natural products. In the current study, we reported profound findings obtained by density functional theory (DFT) simulations, and they were consistent with the experimental conditions. The results set out a catalytic cycle within intermediacy of $\mathrm{NaIO}_{4}$-complexed $\mathrm{Ru}(\mathrm{vl})$ species. Importantly, the co-oxidant played a critical role in the cyclisation step and subsequently the release of THF-diols. Following the formation of $\mathrm{Ru}(\mathrm{VI})$ glycolate, cyclisation and THF-diol release proceeded through $\mathrm{NaIO}_{4}$-coordinated $\mathrm{Ru}(\mathrm{VI})$ intermediates, outpacing the Ru(vIII) glycolate or THF-diolate intermediates and subsequently entering "second cycle" type pathways. The results indicated a cycle involving $\mathrm{Ru}(\mathrm{vIII}) / \mathrm{Ru}(\mathrm{vI}) / \mathrm{Ru}(\mathrm{Iv}) / \mathrm{Ru}(\mathrm{VI})$ rather than $\mathrm{Ru}(\mathrm{VIII}) / \mathrm{Ru}(\mathrm{VI}) / \mathrm{Ru}(\mathrm{VIII}) / \mathrm{Ru}(\mathrm{VI}) / \mathrm{Ru}(\mathrm{VIII})$. Additionally, the existence of an electron-withdrawing group (EWG) on one of the double bonds of 1,5-dienes revealed that the regioselectivity of the Rucatalysed oxidative cyclisation was predominantly initiated at the electron-rich alkene. Overall, this study offers new insights, which were ignored by earlier experimentalists and theoreticians, into the Rucatalysed functionalizations of alkenes and 1,5-dienes.
\end{abstract}

Received 11th March 2020

Accepted 30th March 2020

DOI: $10.1039 / \mathrm{dOra02303e}$

rsc.li/rsc-advances
Previous studies on the $\mathrm{RuO}_{4}$ oxidative cyclisation of 1,5dienes have reported mixtures of cis and trans THF-diol products. ${ }^{11 a}$ However, developments by Stark et al. led to cis-THF diols with high yields and stereoselectivities (Scheme 1). ${ }^{5 \boldsymbol{b}, \mathbf{1 1 b}, \mathbf{1 1 d}}$ They suggested a mechanism analogous to the one presented by Baldwin. ${ }^{9 a}$ It is basically believed that $\mathrm{RuO}_{4}$ interacts with a double bond to form $\mathrm{Ru}(\mathrm{vI})$ glycolate 3 , followed by cyclisation to give THF-diolate Ru(Iv) $\mathbf{4}$ and subsequent hydrolysis to afford cis-THF-diol 2 (Scheme 1).

Mechanistic computational studies on the oxidative cyclisation of 1,5-dienes by metal-oxo species have been reported, but studies concerning Ru-mediated reactions have not been fully reported using potassium permanganate, ${ }^{9}$ which was followed by related stereoselective approaches using osmium and ruthenium..$^{\mathbf{1 0 , 1 1}}$ The first mechanistic proposal was set out for the permanganate-promoted reaction by Walba et al. and was based on the $(2+2)$ suprafacial additions of metal-oxo species across the olefin double bonds. Baldwin proposed an alternative mechanism for Mn oxidative cyclisations based on $(3+2)$ cycloadditions, ${ }^{6 c, 9 a, 12,13}$ which has become a general framework for oxidative cyclisations by $\mathrm{Os}$ and $\mathrm{Ru}$-oxo species, ${ }^{\mathbf{3}, 10,11}$ and the $\mathrm{Ru}$-catalysed version is the concern of this paper. ${ }^{\mathbf{1 4}}$

${ }^{a}$ School of Chemistry, University of Southampton, Southampton, Hampshire, SO17 1BJ, UK. E-mail:aahh1f19@soton.ac.uk

${ }^{b}$ College of Dentistry, University of Al-Ameed, PO Box 198, Karbala, Iraq

$\dagger$ Electronic supplementary information (ESI) available: Additional calculated reaction pathways, cartesian coordinates, absolute energies for reported structures. See DOI: 10.1039/d0ra02303e

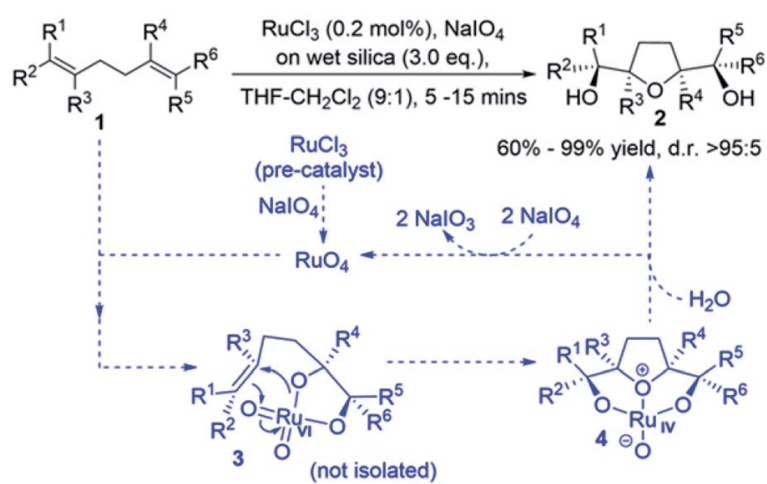

Scheme 1 Ru-mediated oxidative cyclisation of 1,5-diene 1 to afford cis-THF-diol 2. 
investigated. ${ }^{\mathbf{1 3 , 1 5}}$ Despite the reliable use of the reaction in the synthetic methods, ${ }^{\mathbf{1 1}, 14}$ only one step of the catalytic cycle, which is alkene oxidation to yield $\mathrm{Ru}(\mathrm{vI})$ dioxoglycolate, has been studied computationally. ${ }^{\mathbf{1 6}}$ The mechanism of Ru-catalysed oxidative cyclisation involves several steps. These steps are as follows:

(a) alkene oxidation to generate $\mathrm{Ru}(\mathrm{vI})$ glycolate (the first reaction step, FRS); ${ }^{16}$

(b) cyclisation to form the $\mathrm{Ru}(\mathrm{IV})$ THF-diolate (the second reaction step, SRS);

(c) possible secondary reactions that may occur prior to cyclisation such as reoxidation, complexation with a co-oxidant, and hydration with water;

(d) hydrolysis to release the THF-diol; and

(e) other intermolecular $(3+2)$ cycloaddition pathways that propagate the catalytic cycle (Fig. 1).

In this context, almost no details of the mechanism's steps have appeared in the literature. This lack of mechanistic understanding frequently impedes further improvements in the catalytic studies. Therefore, the present study reports a detailed description of these steps, which were ignored by previous researchers, both experimentalists and theoreticians, using computational investigations. Based on the results, we proposed a catalytic cycle and determined the important role of the co-oxidant in the Ru-catalysed oxidative cyclisation of 1,5dienes. Subsequently, the regioselectivity of the reaction was evaluated when an EWG was found on one of the double bonds of 1,5-dienes.

\section{Results and discussion}

The computational approach to the oxidative cyclisation of 1,5diene was performed using DFT simulations with the (SMD/ THF)-M06/aug-cc-pVDZ/LANL2DZ//M06/cc-pVDZ/LANL2DZ level of theory at $298.15 \mathrm{~K}^{17}$ All calculations considered here account for singlet spin multiplicity. ${ }^{18}$ The following sections explain the mechanism of Ru-catalysed oxidative cyclisation and the regioselectivity of the reaction.

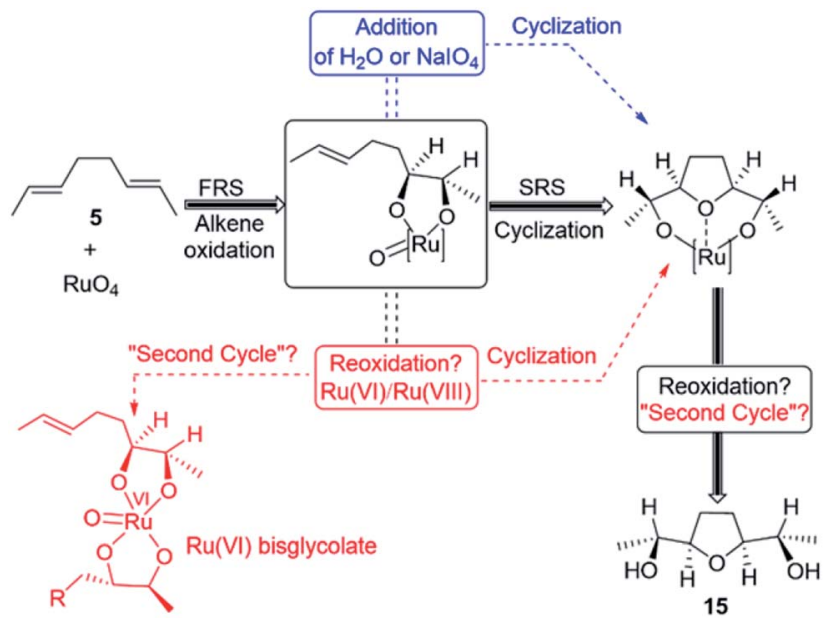

Fig. 1 Pathways obtained for the Ru-catalysed oxidative cyclisation of 1,5-diene (5) in this study.

\section{Mechanism of Ru-catalysed oxidative cyclisation}

The formation of THF-diolate from the oxidative cyclisation of 1,5-diene (5) has been realised through different reaction manifolds considered for SRS after the formation of the $\mathrm{Ru}(\mathrm{vI})$ dioxoglycolate intermediate 7 (Fig. 2). Generally, these manifolds either proceed without or with a co-oxidant $\left(\mathrm{NaIO}_{4}\right)$.

Oxidative cyclisation without a co-oxidant. The simplest pathway to form THF-diolate $\mathbf{9}$ was estimated without considering a co-oxidant (Fig. 2). Initially, the reaction of $\mathrm{RuO}_{4}$ with the double bond of 1,5-diene (5) needed a remarkably low barrier of only $3.2 \mathrm{kcal} \mathrm{mol}^{-1}$ through a tetrahedral $(3+2)$ cycloaddition TS 6 to give a distorted tetrahedral $\mathrm{Ru}(\mathrm{vI})$ ruthenate ester 7 via a highly exergonic step $\left(\Delta G_{\mathrm{r}}=\right.$ $\left.-58.2 \mathrm{kcal} \mathrm{mol}^{-1}\right) \cdot{ }^{19,20}$ Without any additive, this intermediate dynamically cyclised to the square planar $\mathrm{Ru}(\mathrm{Iv})$ diolate $9\left(\Delta G_{\mathrm{r}}=\right.$
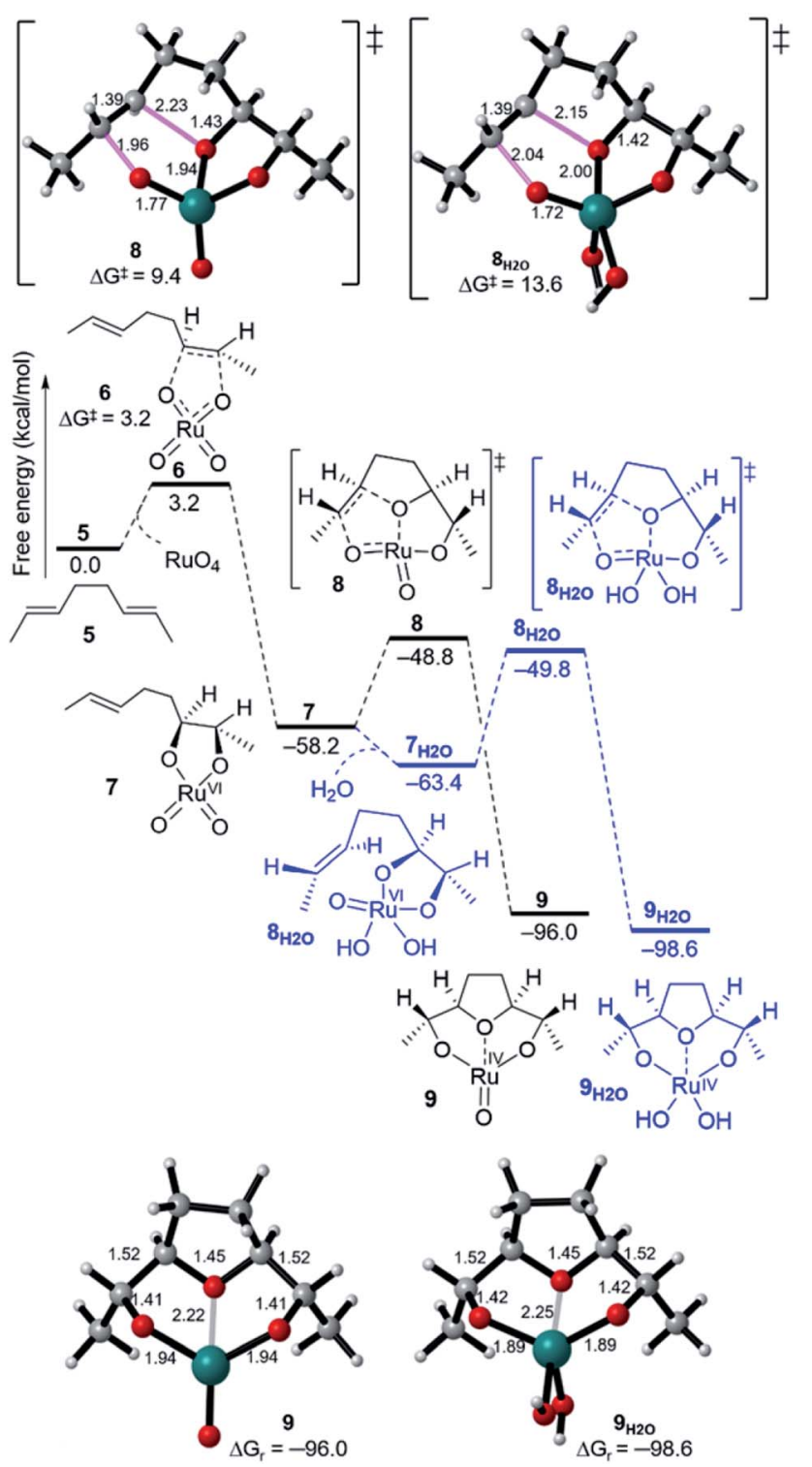

Fig. 2 Free energy profiles for the oxidative cyclisation of 1,5-diene 5 by $\mathrm{RuO}_{4}$, showing cyclisation pathways without water $(7 \rightarrow 9)$ and with water $\left(7_{\mathrm{H} 2 \mathrm{O}} \rightarrow 9_{\mathrm{H} 2 \mathrm{O}}\right)$. 
$\left.-37.8 \mathrm{kcal} \mathrm{mol}^{-1}\right)$ through a tetrahedral $(3+2)$ cycloaddition TS 8 with a barrier of $9.4 \mathrm{kcal} \mathrm{mol}^{-1} \cdot .^{21-23}$ Other possible pathways from $\mathrm{Ru}(\mathrm{vI}) 7$ are the addition of water (blue pathway, Fig. 2) and $\mathrm{NaIO}_{4}$ or reoxidation (Fig. 3).

The addition of a water molecule stabilized $\mathrm{Ru}(\mathrm{vI}) 7$ by $5.2 \mathrm{kcal} \mathrm{mol}^{-1}$ to give a distorted square pyramid $\mathrm{Ru}(\mathrm{vI})$ glycolate $7_{\text {H2O }}$ (Fig. 2, blue pathway). However, this raised the barrier of cyclisation to $13.6 \mathrm{kcal} \mathrm{mol}^{-1}$ via a distorted trigonal bipyramidal $(3+2)$ cycloaddition TS $\mathbf{8}_{\mathbf{H 2 O}}$ to form the trigonal bipyramidal Ru(Iv) diolate $\mathbf{9}_{\mathbf{H} 2 \mathbf{O}}\left(\Delta G_{\mathrm{r}}=-35.2 \mathrm{kcal} \mathrm{mol}^{-1}\right)$. The increased energy barrier can be understood by the effect of the decrease in the electrophilicity of the hydrated $\mathrm{Ru}(\mathrm{VI})$ glycolate $7_{\text {H2O }}$ (see Fig. 4). The LUMO energy of $7_{\text {H2O }}(\mathrm{LUMO}=-3.7 \mathrm{eV}$, $E_{\text {gap }}=3.1 \mathrm{eV}$ ) has been calculated to be higher than that of the non-hydrated $\mathrm{Ru}(\mathrm{vI}) 7\left(\mathrm{LUMO}=-4.2 \mathrm{eV}, E_{\text {gap }}=2.6 \mathrm{eV}\right)$. The HOMO and LUMO orbitals are shown in Fig. 4.

Oxidative cyclisation with a co-oxidant. Now, the addition of $\mathrm{NaIO}_{4}$ to $\mathrm{Ru}(\mathrm{vI}) 7$ forms the $\mathrm{NaIO}_{4}$-complexed $\mathrm{Ru}(\mathrm{vI})$ glycolate $7_{\mathrm{NaIO}}$, which is either cyclised or reoxidized, $\mathrm{Ru}(\mathrm{VI}) \rightarrow \mathrm{Ru}(\mathrm{VIII})$ (see Fig. 3). The effect of reoxidation $\mathrm{Ru}(\mathrm{vI}) \rightarrow \mathrm{Ru}(\mathrm{vIII})$ on oxidative cyclisation was considered first through oxo-ligand transfer from $\mathrm{NaIO}_{4}$ to $\mathrm{Ru}(\mathrm{vI})$ dioxoglycolate (right, Fig. 3). The TS of reoxidation was investigated as two elementary steps, which are $\mathrm{NaIO}_{4}-\mathrm{Ru}(\mathrm{vI})$ complexation and oxo-transfer dissociation, and in an inner sphere manner. ${ }^{2,25}$ The formation of $\mathrm{NaIO}_{4}$-complexed $\mathrm{Ru}(\mathrm{vI}) 7_{\mathrm{NaIO} 4}$ was exergonic by $6.3 \mathrm{kcal} \mathrm{mol}^{-1}$ and preceded the dissociation of the $\mathrm{I}-\mathrm{O}$ bond to release $\mathrm{Ru}(\mathrm{VIII})$ trioxoglycolate and $\mathrm{NaIO}_{3}$ with a high energy barrier of 39.5 kcal mol ${ }^{-1}$ via TS $\mathbf{1 0}$ as an endergonic step $\left(\Delta G_{\mathrm{r}}=\right.$ $16.0 \mathrm{kcal} \mathrm{mol} \mathrm{m}^{-1}$ ). Although the barrier of reoxidation is unfavourable, it was found that once $7_{[\mathbf{o x}]}$ was formed, $\mathrm{Ru}(\mathrm{vI})$ THF-diolate $\mathbf{9}_{[\mathbf{o x}]}$ was directly generated through a barrierless and thermodynamic sink step (TS $8_{[\mathbf{o x}]}, \Delta G^{\ddagger} 0.5$ and $\Delta G_{\mathrm{r}}=$ $-64.5 \mathrm{kcal} \mathrm{mol}^{-1}$ ).

A comparison between $\mathrm{Ru}(\mathrm{VIII})$, hydrated $\mathrm{Ru}(\mathrm{vI})$, and naked $\mathrm{Ru}(\mathrm{vI})$ cyclisation is clearly evident. The $\mathrm{Ru}(\mathrm{vI})$ glycolates 7 and $7_{\text {H2O }}$ have two d electrons, and these can lead to electronic repulsions with the two oxo ligands. ${ }^{26}$ Consequently, this will decrease the electrophilicity and increase the cyclisation barrier. In contrast, this does not occur in $7_{[\mathbf{o x}]}$ and thus, a remarkable increase in the electrophilicity (LUMO $=-4.6 \mathrm{eV}$, $E_{\text {gap }}=2.2 \mathrm{eV}$, Fig. 4) is seen to stimulate barrierless cycloaddition.

It is noteworthy to mention that reoxidation may be competitive when only alkenes are applied and not 1,5-dienes. However, whether the Ru(viII) intermediate is viable during the Ru-catalysed dihydroxylation of alkenes is still disputed. ${ }^{27}$ In this work, DFT simulations showed that reoxidation was excluded due to the high barrier needed. Therefore, there is no possibility of entering a "second cycle" type pathway from the reaction of $7_{[\mathbf{o x}]}$ with another alkene to obtain the $\mathrm{Ru}(\mathrm{Iv})$ bisglycolate (see Fig. 1). ${ }^{4 c, 11 f, 28-30}$

Cyclisation through $\mathrm{NaIO}_{4}$-complexed $\mathrm{Ru}(\mathrm{vI})$ glycolate displayed a low barrier of $2.5 \mathrm{kcal} \mathrm{mol}^{-1}$ via distorted trigonal bipyramidal TS $\mathbf{8}_{\mathrm{NaIO4}}$ and was a favourably exergonic step with $\Delta G_{\mathrm{r}}=-43.1 \mathrm{kcal} \mathrm{mol}^{-1}$ (Fig. 3, right). ${ }^{31}$ When the $\mathrm{NaIO}_{4}$-complexed pathway $\left(7_{\mathbf{N a I O 4}} \rightarrow \mathbf{8}_{\mathrm{NaIO4}} \rightarrow \mathbf{9}_{\mathrm{NaIO4}}\right)$ and the reoxidation pathway $\left(7_{\mathbf{N a I O 4}} \rightarrow 7_{[\mathbf{o x}]} \rightarrow \mathbf{8}_{[\mathbf{o x}]} \rightarrow \mathbf{9}_{[\mathbf{o x}]}\right)$ were compared, the former was exceedingly preferred. Molecular orbital analysis revealed that although the energy gap for $7_{\mathrm{NaIO}}$ was $2.7 \mathrm{eV}$

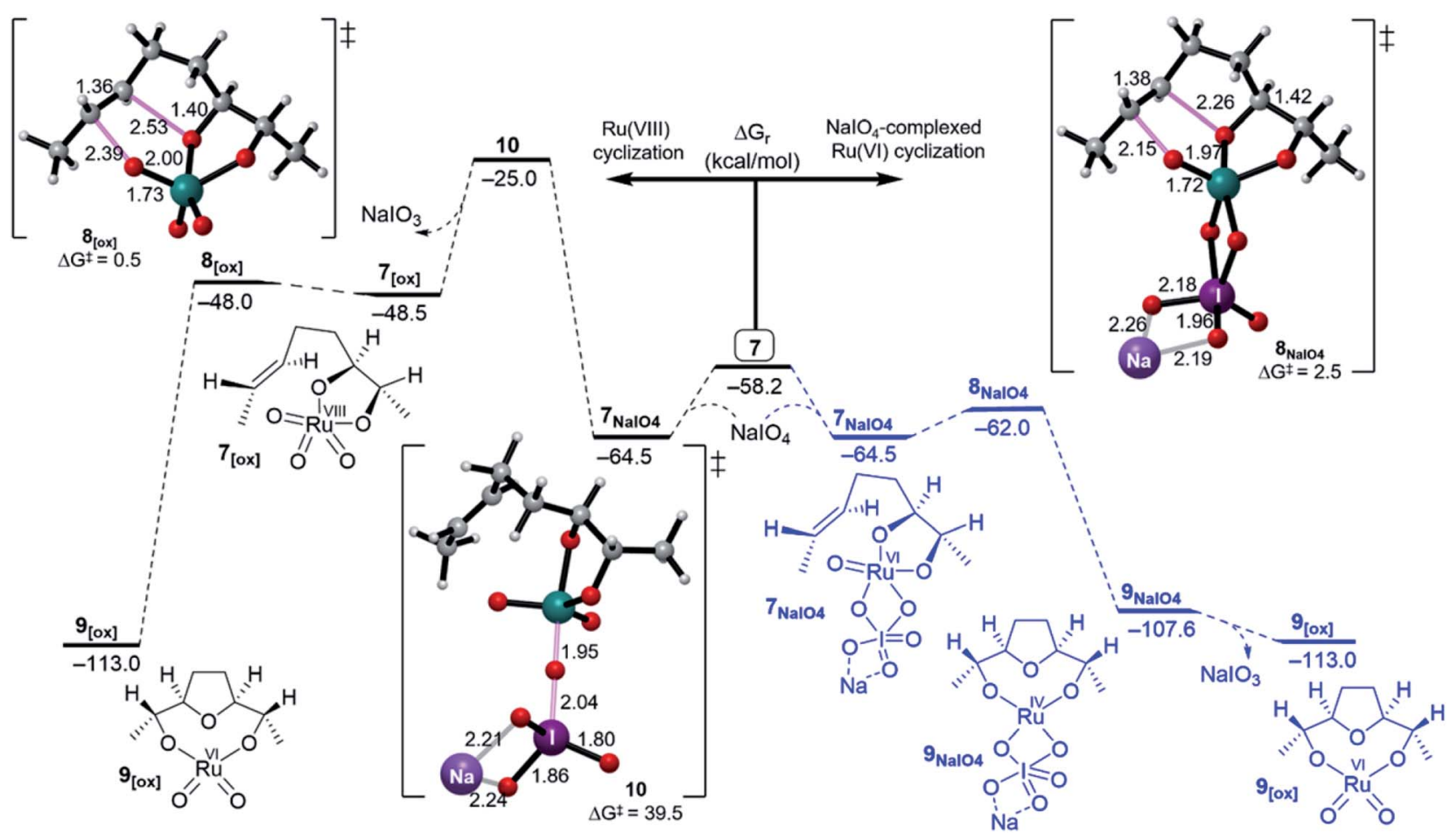

Fig. 3 Free energy profiles for the $\mathrm{Ru}(\mathrm{VIII})$ (right) and $\mathrm{NalO}_{4}$-complexed $\mathrm{Ru}(\mathrm{VI})$ (left) cyclisation pathways. 


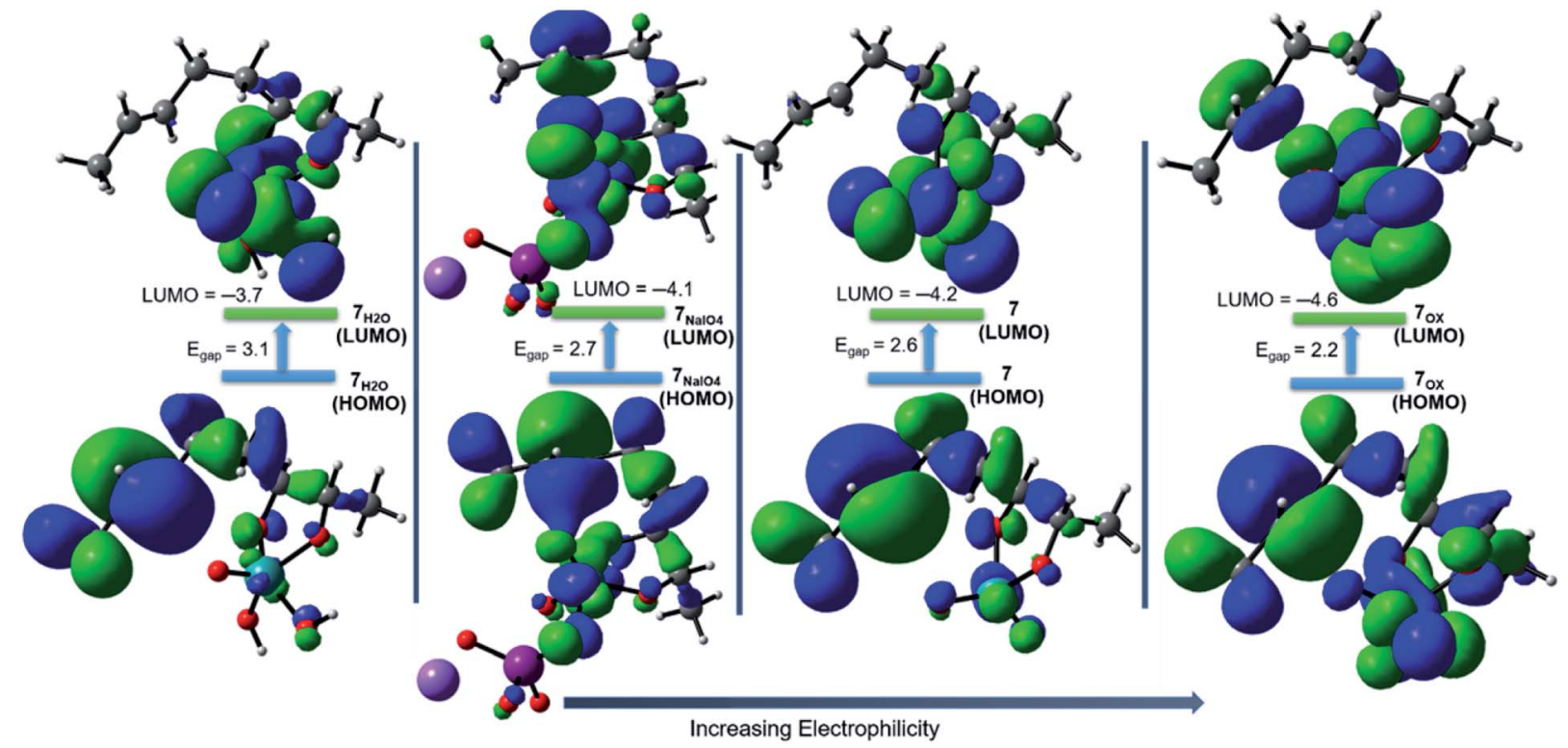

Fig. 4 Visualization (0.02 isovalue surface) of the HOMO (located on the alkene site) and LUMO (located on the Ru site) orbitals of Ru(v) 7, 7 $\mathrm{HzO}_{2}$ and $7_{\mathrm{NalO} 4}$ and $\mathrm{Ru}(\mathrm{VIII}) 7_{\text {[ox] }}$ glycolates during the oxidative cyclisation of 1,5-diene (5) by $\mathrm{RuO}_{4}$.

(Fig. 4), which was slightly lower than that for 7, its TS $\mathbf{8}_{\mathbf{N a I O 4}}$ had a significantly lower barrier. This may be attributed to the favourable interactions between the reacting moieties with less geometrical changes or reorganizations around the $\mathrm{Ru}$ centre due to the exchangeable oxo ligands between the I and $\mathrm{Ru}$ centres that lead to a more stabilized TS to give Ru(Iv) THF-

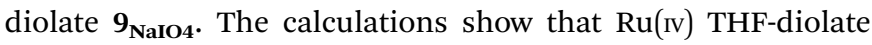
$\mathbf{9}_{\mathrm{NaIO}}$ is simultaneously dissociated to $\mathrm{Ru}(\mathrm{vI})$ dioxodiolate $\mathbf{9}_{\text {[ox] }}$ in a favoured step $\left(\Delta G_{\mathrm{r}}=-6.6 \mathrm{kcal} \mathrm{mol}^{-1}\right)$ (Fig. 3, right).

In summary, in the presence of $\mathrm{NaIO}_{4}$, cyclisation through $\mathrm{NaIO}_{4}$-coordinated $\mathrm{Ru}(\mathrm{vI})$ glycolate $7_{\mathrm{NaIO}}$ outpaces cyclisation via hydrated $\mathrm{Ru}(\mathrm{vI}) 7_{\mathbf{H} 2 \mathrm{O}}$ and reoxidized $\mathrm{Ru}(\mathrm{VIII}) 7_{[\mathbf{o x}]}$. In this regard, the simulations showed that reoxidation was energetically unfavourable and thus, the formation of a "second cycle" type pathway product, $\mathrm{Ru}(\mathrm{vI})$ bisglycolate, was totally outpaced.

THF-diol release. The calculations reported above support the cyclisation of periodate-complexed $\mathrm{Ru}(\mathrm{vI})$ to give an $\mathrm{Ru}(\mathrm{IV})$ THF-diolate product. The completion of a catalytic cycle to furnish THF-diol 15 and regenerate a Ru(VIII) species capable of propagating ruthenylation is important. Now, the $\mathrm{Ru}(\mathrm{vI})$ dioxodiolate $9_{[\mathbf{o x}]}$ entails pathways toward either oxidation/hydrolysis or hydrolysis/oxidation while bearing in mind that $\mathbf{9}_{\text {[ox] }}$ can engage in the ruthenylation of another 1,5-diene molecule (Fig. 5).

First, the oxidation of Ru(Iv) THF-dioxodiolate $\mathbf{9}_{[\mathbf{o x}]}$ to $\mathrm{Ru}(\mathrm{VIII})$ trioxodiolate $\mathbf{9}_{[\mathbf{o x}][\mathbf{o x}]}$ is considered with an overall endergonicity of $13.5 \mathrm{kcal} \mathrm{mol}^{-1}$ (Fig. 5, blue pathway). ${ }^{32}$ In this regard, $\mathrm{Ru}(\mathrm{VIII})$ THF-trioxodiolate $\mathbf{9}_{[\mathbf{o x}][\mathbf{o x}]}$ can overcome a barrier of $21.1 \mathrm{kcal} \mathrm{mol}^{-1}$ via TS 12 to break the first $\mathrm{O}-[\mathrm{Ru}]$ diolate in a modestly exergonic step $\left(\Delta G_{\mathrm{r}}=-6.5 \mathrm{kcal} \mathrm{mol}^{-1}\right.$, see 13, Fig. 5), which is followed by the release of THF-diol 15 as an overall exergonic hydrolysis pathway $\left(\Delta G_{\mathrm{r}}=-20.9 \mathrm{kcal} \mathrm{mol}^{-1}\right)$. Instead, DFT simulations indicate that $\mathrm{Ru}(\mathrm{vI})$ THF-dioxodiolate $\mathbf{9}_{\text {[ox] }}$ coordinates with $\mathrm{NaIO}_{4}$ and then $\mathrm{H}_{2} \mathrm{O}$ to yield 16 via a low endergonic step $\left(\Delta G_{\mathrm{r}}=3.4 \mathrm{kcal} \mathrm{mol}{ }^{-1}\right.$, Fig. 5). Also, 16 needs a comparatively low barrier of $10.7 \mathrm{kcal} \mathrm{mol}^{-1}$ via a TS 17 to give intermediate 18 via an exergonic process $\left(\Delta G_{\mathrm{r}}=\right.$ $-3.4 \mathrm{kcal} \mathrm{mol}^{-1}$ ). In the opposite mode to the hydrolysis of $\mathrm{Ru}(\mathrm{VIII})$, the hydrolysis of the second $\mathrm{O}-[\mathrm{Ru}]$ diolate in intermediate 18 was calculated to be unfavourable when compared to the experimental conditions. The located TS 22, shown in ESI Fig. $4, \dagger$ requires a high energy barrier of $21.7 \mathrm{kcal} \mathrm{mol}^{-1}$ to transfer the proton from the oxo ligand ( $\mathrm{HO}-[\mathrm{Ru}])$ to the second $\mathrm{O}-[\mathrm{Ru}]$ diolate bond although it is an exergonic step $\left(\Delta G_{\mathrm{r}}=\right.$ $\left.-7.6 \mathrm{kcal} \mathrm{mol}^{-1}\right)$. Obviously, proton transfers involving electronegative atoms are known to be fast and diffusion controlled and therefore, getting intermediate 19 via a slightly endergonic step $\left(\Delta G_{\mathrm{r}}=6.3 \mathrm{kcal} \mathrm{mol}^{-1}\right)$ is suggested. At this point, hydrolysis through $\mathrm{Ru}(\mathrm{vI})$ followed by oxidation to regenerate $\mathrm{RuO}_{4}$ is more accessible than reoxidation, i.e., $\mathrm{Ru}(\mathrm{vI}) \rightarrow \mathrm{Ru}(\mathrm{VIII})$ followed by hydrolysis.

Second, it is interesting to note that $\mathrm{Ru}(\mathrm{vI})$ dioxodiolate $\mathbf{9}_{\text {[ox] }}$ is a competent ruthenylating agent in the hydrolysis pathway and is capable of reacting with another alkene via a so-called "second cycle" type pathway (Fig. 5, left pathway). ${ }^{33}$ The energy barrier of the $(3+2)$ cycloaddition of $9_{[\mathbf{o x}]}$ with $E$-but-2ene was found to be $15.4 \mathrm{kcal} \mathrm{mol}^{-1}$ via TS 19 to afford a tetraester 20 via an exergonic reaction $\left(\Delta G_{\mathrm{r}}=-50.5 \mathrm{kcal} \mathrm{mol}^{-1}\right)$. This would reveal a barrier difference of $1.3 \mathrm{kcal} \mathrm{mol}^{-1}$ between intermolecular ruthenylation and hydrolysis with the hydrolysis being predominant.

Finally, based on the above-mentioned results, a catalytic cycle for the Ru-catalysed oxidative cyclisation of 1,5-diene 5 was proposed (Fig. 6). The complexation of $\mathrm{Ru}(\mathrm{vI})$ glycolate 7 with a co-oxidant $\left(\mathrm{NaIO}_{4}\right)$ is an essential feature found for SRS. The reoxidation of $\mathrm{NaIO}_{4}$-coordinated $\mathrm{Ru}(\mathrm{IV})$ THF-diolate $\mathbf{9}_{\mathbf{N a I O 4}}$ to $\mathrm{Ru}(\mathrm{vI}) \mathrm{THF}$-dioxodiolate $\mathbf{9}_{[\mathbf{o x}]}$ followed by another coordination 


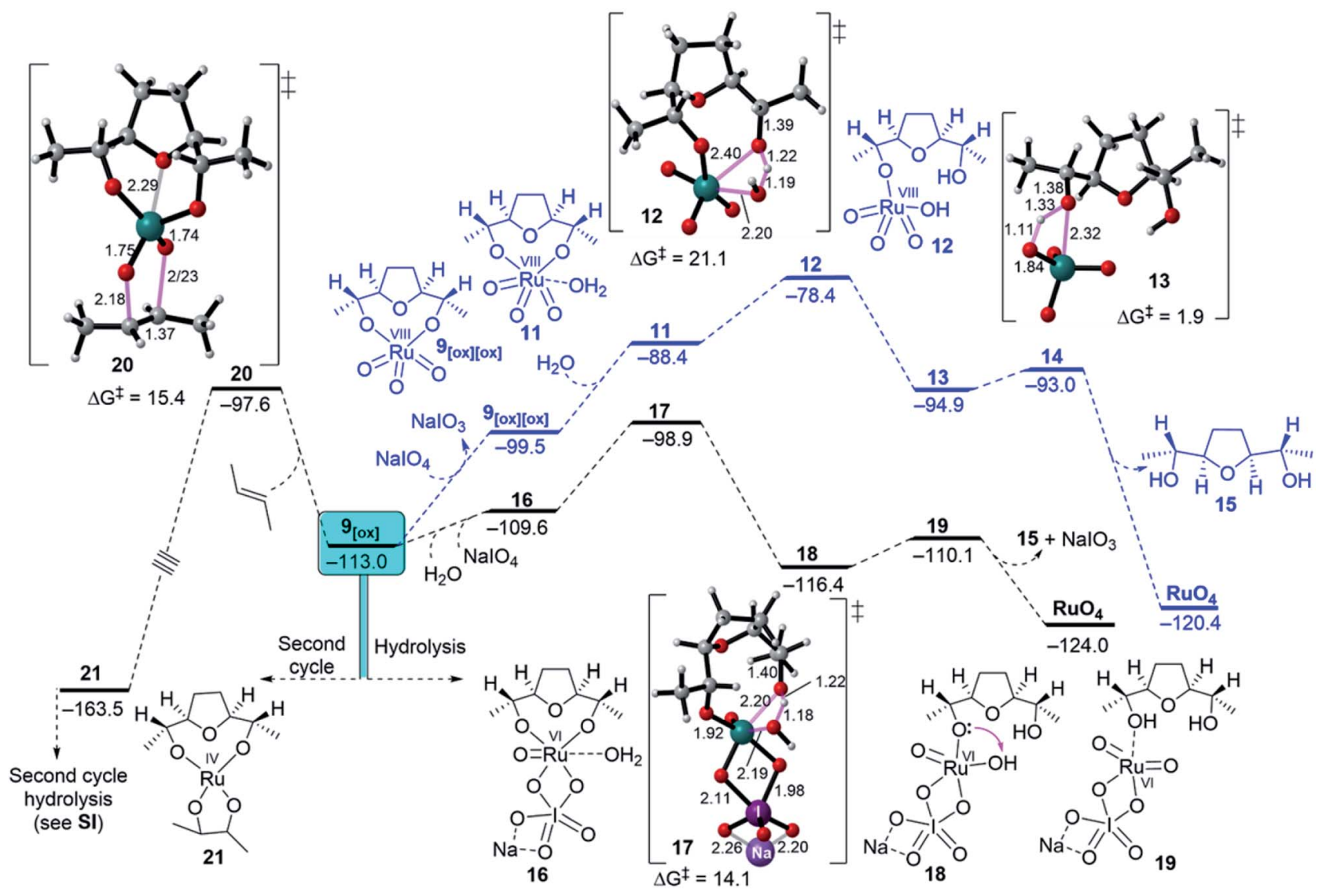

Fig. 5 Free energy evaluations for divergent reactions of $9_{\text {[ox] }}$, showing hydrolysis via Ru(VI) or Ru(VIII) or entering a second cycle through another ruthenylation. E-But-2-ene was used as a model for 5 to reduce the computational cost.

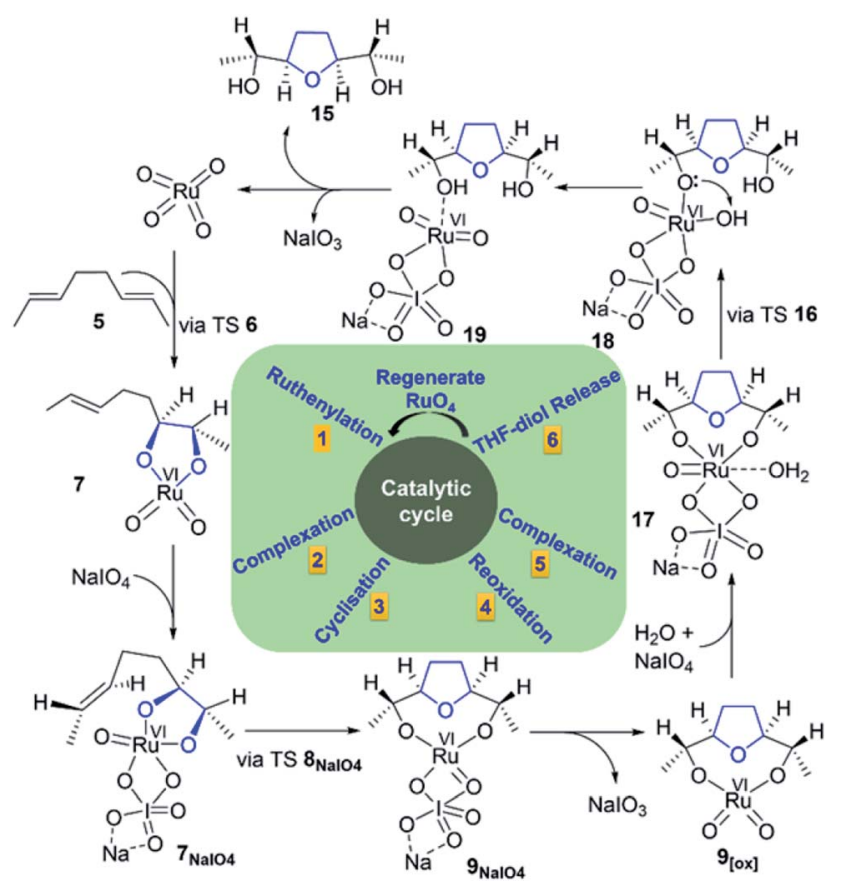

Fig. 6 Proposed catalytic cycle for Ru-catalysed oxidative cyclisation of 1,5-diene 5 . with both water and $\mathrm{NaIO}_{4}$ gives 17, releasing its THF-diol 15 and regenerating $\mathrm{RuO}_{4}$ to continue the iterative cycle.

\section{Regioselectivity of the Ru-catalysed oxidative cyclisation}

The regioselectivity of $\mathrm{RuO}_{4}$ is considered when an EWG such as a methyl ester group is found on one of the double bonds of 1,5diene, as indicated in methyl dienoate 5a shown in Fig. 7.11b Based on the aforementioned findings, DFT calculations have been performed for FRS and SRS (Fig. 7). For FRS, the favourability of $\mathrm{RuO}_{4}$ to initiate the electron-rich alkene (ERA) pathway over the electron-deficient alkene (EDA) pathway is highly evident. For instance, the ERA Ru(vi) glycolate 7a $\left(\Delta G_{\mathrm{r}}=\right.$ $\left.-62.4 \mathrm{kcal} \mathrm{mol}^{-1}\right)$ is formed via a barrierless $(3+2)$ cycloaddition TS 6 a of $\Delta G^{\ddagger}=1.7 \mathrm{kcal} \mathrm{mol}^{-1}$ (Fig. 7, right), whereas the formation of the EDA $\mathrm{Ru}(\mathrm{VI})$ glycolate $\mathbf{d}-7 \mathbf{a}$ requires a higher barrier of $4.9 \mathrm{kcal} \mathrm{mol}^{-1}$ via TS d-6a via a slightly less exergonic step $\left(\Delta G_{\mathrm{r}}=-55.1 \mathrm{kcal} \mathrm{mol}^{-1}\right.$ (Fig. 7, left). ${ }^{34}$ Thus, the ERA product is kinetically $\left(\Delta \Delta G^{*}=-3.2 \mathrm{kcal} \mathrm{mol}^{-1}\right)$ and thermodynamically $\left(\Delta \Delta G_{\mathrm{r}}=-7.3 \mathrm{kcal} \mathrm{mol}^{-1}\right)$ favoured with a greater development of the $\mathrm{C}-\mathrm{O}$ bond formation for ERA TS 6 a over EDA TS d-6a.

On the cyclisation step, the ERA Ru(vi) glycolate $7 \mathbf{a}_{\text {NaIO4 }}$ cyclises, via TS $8 \mathbf{a}_{\mathbf{N a I O}}$ with a barrier of $\Delta G^{*}=8.6 \mathrm{kcal} \mathrm{mol}^{-1}$, and this is slower than cyclisation through EDA Ru(vI) glycolate 


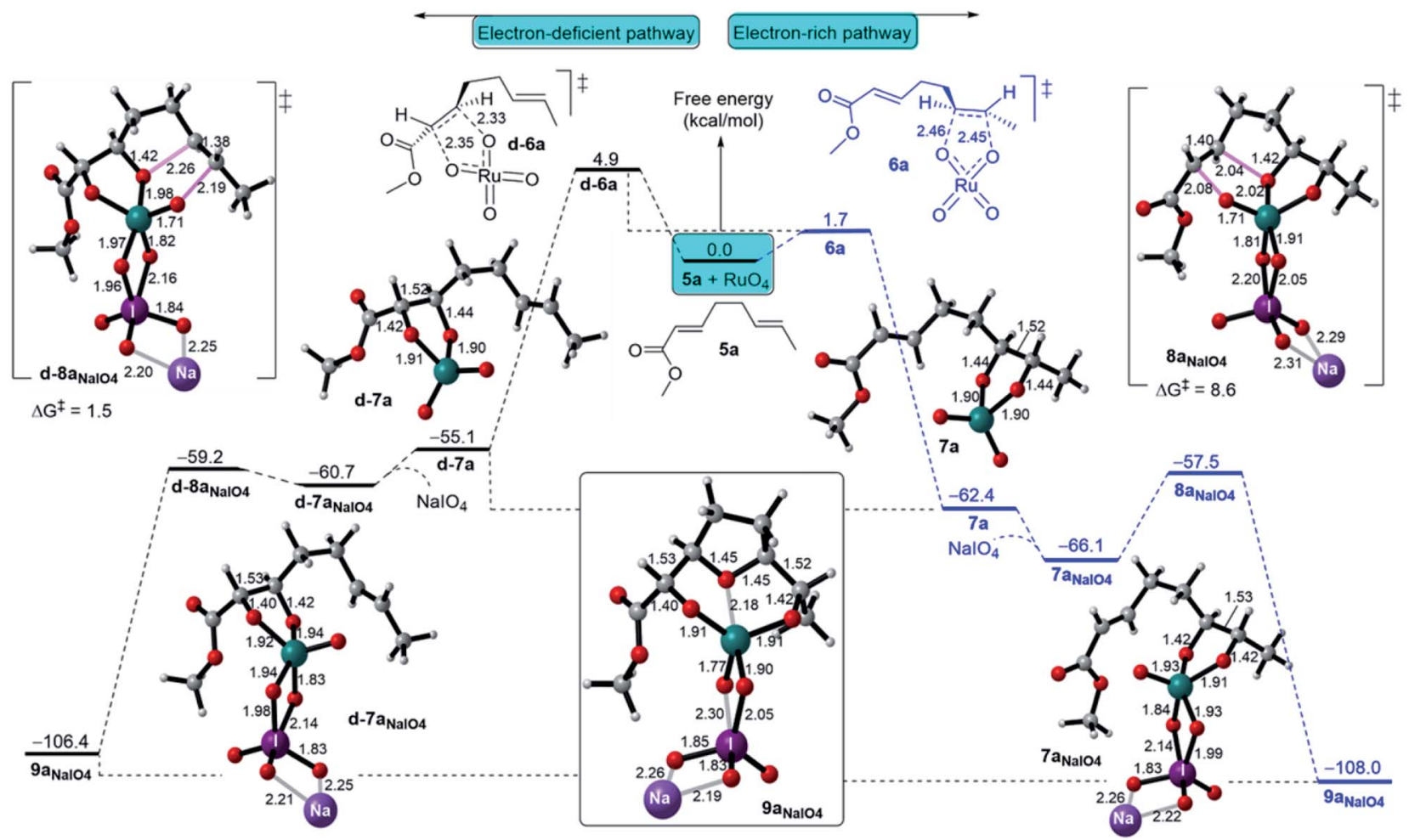

Fig. 7 Free energy bifurcation for the regioselectivity of the Ru-catalyzed oxidative cyclisation of methyl 1,5-dienoate (5a) initiated at the electron-rich and electron-deficient alkenes.

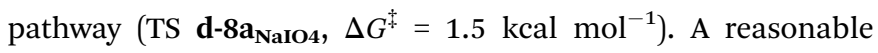
explanation for this is that the oxo ligands in $\mathbf{d}-7 \mathbf{a}_{\mathrm{NaIO}}$ are more electrophilic than those in $\mathbf{7} \mathbf{a}_{\mathrm{NaIO}}$ mainly due to the electronwithdrawing nature of the carbonyl group with a possible minor contribution from complexation with $\mathrm{NaIO}_{4}$. This would lead to a lower barrier for cyclisation because it has lower LUMO energy on the Ru site and higher HOMO energy on the alkene site. However, in the ERA Ru(vI) glycolate $7 \mathbf{a}_{\mathbf{N a I O} 4}$, cyclisation occurs from the highly electron-deficient alkene site to the slightly electron-deficient $\mathrm{Ru}$ site, subsequently ensuring that the cyclisation has a higher barrier. In comparison with the symmetric 1,5-diene (5), the cyclisation via the ERA $\mathrm{NaIO}_{4}$ complexed $\mathrm{Ru}(\mathrm{VI})$ glycolate requires a three-fold higher energy barrier than the cyclisation with the symmetric 1,5-diene (5). Overall, the existence of an EWG on one of the alkenes in the 1,5-diene structure results in the Ru-catalysed oxidative cyclisation being predominantly initiated at the electron-rich alkene.

\section{Conclusions}

The first detailed mechanistic study on the Ru-catalysed oxidative cyclisation of 1,5-dienes has been performed with the (SMD/THF)-M06/aug-cc-pVDZ/LANL2DZ//M06/cc-pVDZ/ LANL2DZ level of theory. The DFT simulations are in excellent agreement with the experimental conditions and consequently, complications accompanied throughout the Ru-catalysed oxidative cyclisation of 1,5-dienes are resolved.
The results set out the catalytic cycle for THF-diol formation through the intermediacy of $\mathrm{Ru}(\mathrm{vI})$ with $\mathrm{RuO}_{4}$ as the active catalytic species. Importantly, the co-oxidant $\mathrm{NaIO}_{4}$ has been evidently shown to play a critical role in the cyclisation and hydrolysis steps through complexation with $\mathrm{Ru}$ rather than reoxidation to a higher oxidation state. Following the initially formed $\mathrm{Ru}(\mathrm{vI})$ dioxoglycolate, the cyclisation overcomes a favourable $\mathrm{NaIO}_{4}$-coordinated TS to give the THF-diolate ring, excluding the cyclisation or entering the second cycle through the intermediacy of $\mathrm{Ru}(\mathrm{viII})$ trioxoglycolate $7_{[\mathbf{o x}]}$. Thereafter, the dissociation of the $\mathrm{NaIO}_{4}$-coordinated $\mathrm{Ru}(\mathrm{Iv})$ THF-diolate $\mathbf{9}_{\mathrm{NaIO}}$ to $\mathrm{Ru}(\mathrm{vI})$ THF-dioxodiolate $\mathbf{9}_{[\mathrm{ox}]}$ was calculated to undergo hydrolytic release to give THF-diol 15 and $\mathrm{RuO}_{4}$ to continue the iterative cycle. The release of THF-diol 15 from Ru(vi) THFdioxodiolate $9_{[\mathrm{ox}]}$ was found to be favourable over reoxidation to $\mathrm{Ru}(\mathrm{VIII})$ THF-trioxodiolate $\mathbf{9}_{[\mathbf{o x}][\mathbf{o x}]}$ or entering the so-called "second cycle" type pathway. This protocol has evidently conducted a cycle of $\mathrm{Ru}(\mathrm{vIII}) / \mathrm{Ru}(\mathrm{vI}) / \mathrm{Ru}(\mathrm{Iv}) / \mathrm{Ru}(\mathrm{vI})$ rather than $\mathrm{Ru}(\mathrm{VIII}) /$ $\mathrm{Ru}(\mathrm{vI}) / \mathrm{Ru}(\mathrm{VIII}) / \mathrm{Ru}(\mathrm{vI}) / \mathrm{Ru}(\mathrm{VIII})$.

Furthermore, DFT simulations on asymmetric 1,5-dienes, such as methyl 1,5-dienoate (5a), have provided further mechanistic descriptions when an electron-withdrawing group is attached to one of the alkenes. The calculations revealed that the Ru-catalysed oxidative cyclisation is predominantly initiated at the electron-rich alkene. The cyclisation from the ERA $\mathrm{NaIO}_{4^{-}}$ complexed $\mathrm{Ru}(\mathrm{vr})$ glycolate was shown to be slower than the cyclisation from an electron-deficient $\mathrm{Ru}(\mathrm{vI})$ intermediate. In comparison with symmetric 1,5-diene (5), the barrier for 
cyclisation from an asymmetric 1,5-diene was calculated to be more than three times higher than the barrier for symmetric cyclisation.

Overall, this computational study gives significantly important insights into this key synthetic method, which may offer a viable pathway towards advancing an efficient protocol for alkene oxidation.

\section{Computational details}

All calculations were performed using Gaussian 09 (ref. 35) with the global hybrid functional approximations M06 (ref. 36) used with the augmented correlation-consistent polarized valence double- $\zeta$ (DZ) basis set (cc-pVDZ and aug-cc-pVDZ) ${ }^{37}$ for $\mathrm{C}, \mathrm{H}, \mathrm{O}, \mathrm{Si}$, and $\mathrm{Na}$, whereas an effective core potential basis step LANL2DZ ${ }^{38}$ was used for Ru and I. All geometry optimisations, even for triplet and quintet spin multiplicities, shown in the ESI, $\dagger$ were performed with the basis set cc-pVDZ/ LANL2DZ, in which all minima intermediates were verified by the absence of negative eigenvalues in the vibrational frequency analysis. Restricted spin Hartree-Fock calculations were used for singlet spin multiplicity, whereas unrestricted spin calculations were used for triplet and quintet spin multiplicity. All of the transition state structures were found using the Berny algorithm, ${ }^{39}$ verified by vibrational analysis, and visualised by animating the negative eigenvector coordinate. In order to account for the effect of the solvent, singlepoint energies of the optimised geometries were evaluated with the M06/aug-cc-pVDZ/LANL2DZ level of theory in tetrahydrofuran (THF) as a representative solvent medium via the solvation model based on density (IEFPCM-SMD). ${ }^{40}$ To obtain the free energies at $298.15 \mathrm{~K}$ and $1 \mathrm{~atm}$, the thermal corrections were evaluated from the unscaled vibrational frequencies at the M06/cc-pVDZ/LANL2DZ level of theory and were then added to the electronic energies calculated from the M06/ aug-cc-pVDZ/LANL2DZ level of theory. Intrinsic reaction coordinate (IRC) calculations were performed for the identified transition states to confirm the reaction path proceeding in both directions (reactant and product), in which the Hessian was recomputed every 3 predictor steps with a step size along the reaction path of 0.05 Bohr. ${ }^{41}$ All activation free energies are quoted relative to infinitely separated reagents and the optimised structures were illustrated using CYLview. ${ }^{\mathbf{4 2}}$

\section{Conflicts of interest}

There are no conflicts to declare.

\section{Acknowledgements}

I acknowledge the computational resources from the iridis4 supercomputer supported by the University of Southampton. I highly acknowledge the University of Southampton/School of Chemistry for providing the visitor-status research position (2717441/EB00-VISIT). Many thanks to Prof. Richard C. D. Brown for his valuable proofreading and his precious support.

\section{Notes and references}

1 For reviews of THF-containing natural products see. Polyether ionophores: (a) J. Rutkowski and B. Brzezinski, Structures and Properties of Naturally Occurring Polyether Antibiotics, BioMed Res. Int., 2014, 21, 1559-1581; (b) A. Huczynski, Polyether ionophores-promising bioactive molecules for cancer therapy, Bioorg. Med. Chem. Lett., 2012, 22, 7002-7010; (c) D. A. Kevin, D. A. F. Meujo and M. T. Hamann, Polyether ionophores: broad-spectrum and promising biologically active molecules for the control of drug-resistant bacteria and parasites, Expert Opin. Drug Discovery, 2009, 4, 109-146; (d) C. J. Dutton, B. J. Banks and C. B. Cooper, Polyether ionophores, Nat. Prod. Rep., 1995, 12, 165-181; (e) A. Acetogenins, R. Tundis, J. B. Xiao and M. R. Loizzo, Annona species (Annonaceae): a rich source of potential antitumor agents?, Ann. N. Y. Acad. Sci., 2017, 1398, 30-36; (f) C. C. Liaw, J. R. Liou, T. Y. Wu, F. R. Chang and Y. C. Wu, Acetogenins from Annonaceae, Prog. Chem. Org. Nat. Prod., 2016, 101, 113-230; (g) A. Bermejo, B. Figadere, M. C. Zafra-Polo, I. Barrachina, E. Estornell and D. Cortes, Acetogenins from Annonaceae: recent progress in isolation, synthesis and mechanisms of action, Nat. Prod. Rep., 2005, 22, 269-303; (h) M. Leboeuf, A. Cavé, P. K. Bhaumik, B. Mukherjee and R. Mukherjee, The phytochemistry of the annonaceae, Phytochemistry, 1980, 21, 2783-2813THF-containing macrolides: (i) A. Lorente, J. Lamariano-Merketegi, F. Albericio and M. Alvarez, Tetrahydrofuran-containing macrolides: a fascinating gift from the deep sea, Chem. Rev., 2013, 113, 4567-4610.

2 For reviews of THF synthesis see: (a) A. de la Torre, C. Cuyamendous, V. Bultel-Ponce, T. Durand, J. M. Galano and C. Oger, Recent advances in the synthesis of tetrahydrofurans and applications in total synthesis, Tetrahedron, 2016, 72, 5003-5025; (b) J. D. Rainier, Synthesis of Substituted Tetrahydrofurans. In Synthesis of Saturated Oxygenated Heterocycles I: 5- and 6-Membered Rings, Top. Heterocycl. Chem., 2014, 35, 1-41; (c) G. Jalce, $\mathrm{X}$. Franck and B. Figadère, Diastereoselective synthesis of 2,5-disubstituted tetrahydrofurans, Tetrahedron: Asymmetry, 2009, 20, 2537-2581; (d) J. P. Wolfe and M. B. Hay, Recent advances in the stereoselective synthesis of tetrahydrofurans, Tetrahedron, 2007, 63, 261-290; (e) M. C. Elliott, Saturated oxygen heterocycles, J. Chem. Soc., Perkin Trans. 1, 2002, 2301-2323; (f) M. M. Faul and B. E. Huff, Strategy and methodology development for the total synthesis of polyether ionophore antibiotics, Chem. Rev., 2000, 100, 2407-2473; (g) T. L. B. Boivin, Synthetic routes to tetrahydrofuran, tetrahydropyran, and spiroketal units of polyether antibiotics and a survey of spiroketals of other natural-products, Tetrahedron, 1987, 43, 3309-3362.

3 For further examples see: (a) A. Roushanbakhti, Y. F. Liu, P. C. M. Winship, M. J. Tucker, W. M. Akhtar, D. S. Walter, G. Wrigley and T. J. Donohoe, Cobalt versus Osmium: Control of Both trans and cis Selectivity in Construction of 
the EFG Rings of Pectenotoxin 4, Angew. Chem., Int. Ed., 2017, 56, 14883-14887; (b) N. S. Sheikh, Synthetic endeavours towards oxasqualenoid natural products containing 2,5-disubstituted tetrahydrofurans-eurylene and teurilene, Nat. Prod. Rep., 2014, 31, 1088-1100; (c) T. J. Donohoe and R. M. Lipiński, Interplay of Cascade Oxidative Cyclisation and Hydride Shifts in the Synthesis of the ABC Spiroketal Ring System of Pectenotoxin-4, Angew. Chem., Int. Ed., 2013, 52, 2491-2494; (d) H. Göksel and C. B. W. Stark, Total Synthesis of cis-Solamin: Exploiting the $\mathrm{RuO}_{4}$-Catalysed Oxidative Cyclisation of Dienes, Org. Lett., 2006, 8, 3433-3436; (e) B. Lygo, D. Slack and C. Wilson, Synthesis of neodysiherbaine, Tetrahedron Lett., 2005, 46, 6629-6632.

4 For reviews see: (a) J. Adrian, L. J. Gross and C. B. W. Stark, The direct oxidative diene cyclisation and related reactions in natural product synthesis, Beilstein J. Org. Chem., 2016, 12, 2104-2123; (b) N. S. Sheikh, Comparative perspective and synthetic applications of transition metal mediated oxidative cyclisation of 1,5-dienes towards cis-2,5disubstituted tetrahydrofurans, Org. Biomol. Chem., 2014, 12, 9492-9504; (c) V. Piccialli, Ruthenium tetroxide and perruthenate chemistry. Recent advances and related transformations mediated by other transition metal oxospecies, Molecules, 2014, 19, 6534-6582; (d) B. S. Pilgrim and T. J. Donohoe, Osmium-catalysed oxidative cyclisation of dienes and their derivatives, J. Org. Chem., 2013, 78, 2149-2167; (e) V. Piccialli, Oxidative cyclisation of dienes and polyenes mediated by transition-metal-oxo species, Synthesis, 2007, 2585-2607.

5 For other oxidative cyclisations of 1,4-dienes, 1,6-dienes and 1,7-dienes see: (a) V. Piccialli, N. Borbone and G. Oliviero, Ruthenium-catalysed oxidative cyclisation of 1,7-dienes. A novel diastereoselective synthesis of 2,7-disubstituted transoxepane diols, Tetrahedron Lett., 2007, 48, 5131-5135; (b) S. Roth and C. B. W. Stark, Efficient oxidative cyclisation of 1,6-dienes: a highly diastereoselective entry to substituted tetrahydropyrans, Angew. Chem., Int. Ed., 2006, 45, 62186221; (c) A. R. L. Cecil and R. C. D. Brown, Stereoselective synthesis of cis-2,6-bis-hydroxyalkyl-tetrahydropyrans by the permanganate promoted oxidative cyclisation of 1,6-dienes, Tetrahedron Lett., 2004, 45, 7269-7271; (d) B. Travis and B. Borhan, Oxidative cyclisation of 1,4-dienes to yield 2,3,5trisubstituted tetrahydrofuran-diols, Tetrahedron Lett., 2001, 42, 7741-7745; (e) V. Piccialli, $\mathrm{RuO}_{4}$-catalysed oxidative cyclisation of 1,6-dienes to trans-2,6bis(hydroxymethyl)tetrahydropyranyldiols. A novel stereoselective process, Tetrahedron Lett., 2000, 41, 37313733.

6 (a) A. M. Al Hazmi, N. S. Sheikh, C. J. R. Bataille, A. A. M. AlHadedi, S. V. Watkin, T. J. Luker, N. P. Camp and R. C. D. Brown, trans-2-Tritylcyclohexanol as a chiral auxiliary in permanganate-mediated oxidative cyclisation of 2-methylenehept-5-enoates: application to the synthesis of trans-(+)-linalool oxide, Org. Lett., 2014, 16, 5104-5107; (b) R. C. D. Brown and P. J. Kocienski, A synthesis of salinomycin 1. Synthesis of key fragments, Synlett, 1994,
415-417; (c) D. M. Walba, M. D. Wand and M. C. Wilkes, Stereochemistry of the permanganate oxidation of 1,5dienes, J. Am. Chem. Soc., 1979, 101, 4396-4397.

7 R. C. D. Brown and J. F. Keily, Asymmetric permanganatepromoted oxidative cyclisation of 1,5-dienes by using chiral phase-transfer catalysis, Angew. Chem., Int. Ed., 2001, 40, 4496-4498.

8 For cis-selective THF-diol formation from oxidative cyclisation of dihydroxyalkenes using metal oxo agents $(\mathrm{Ru}$, Os, and Cr). For Ru oxo-species: (a) H. Cheng and C. B. W. Stark, A double donor-activated ruthenium(VII) catalyst: synthesis of enantiomerically pure THF-diols, Angew. Chem., Int. Ed., 2010, 49, 1587-1590; (b) S. Gohler and C. B. W. Stark, Catalytic diastereo- and position selective oxidative mono-cyclisation of 1,5,9-trienes and polyenes, Org. Biomol. Chem., 2007, 5, 1605-1614Os oxospecies: (c) T. J. Donohoe, K. M. P. Wheelhouse, P. J. Lindsay-Scott, P. A. Glossop, I. A. Nash and J. S. Parker, Pyridine- $N$-oxide as a mild reoxidant which transforms osmium-catalysed oxidative cyclisation, Angew. Chem., Int. Ed., 2008, 47, 2872-2875; (d) T. J. Donohoe and S. Butterworth, Oxidative cyclisation of diols derived from 1,5-dienes: formation of enantiopure cis-tetrahydrofurans by using catalytic osmium tetroxide; Formal synthesis of (+)-cis-solamin, Angew. Chem., Int. Ed., 2005, 44, 47664768Cr oxo-species: (e) E. J. Corey and D. C. Ha, Total synthesis of venustatriol, Tetrahedron Lett., 1988, 29, 31713174; (f) D. M. Walba and G. S. Stoudt, Oxidative cyclisation of 5,6-dihydroxyalkenes promoted by $\mathrm{Cr}(\mathrm{VI})$ oxo species: A novel cis-2,5-disubstituted tetrahydrofuran synthesis, Tetrahedron Lett., 1982, 23, 727-730; $(g)$ B. D. Hammock, S. S. Gill and J. E. Casida, Synthesis and morphogenetic activity of derivatives and analogs of aryl geranyl ether juvenoids, J. Agric. Food Chem., 1974, 22, 379385.

9 (a) J. E. Baldwin, M. J. Crossley and E.-M. M. Lehtonen, Stereospecificity of oxidative cycloaddition reactions of 1,5dienes, J. Chem. Soc., Chem. Commun., 1979, 918-920; (b) E. Klein and W. Rojahn, Permanganate oxidation of 1,5diene compounds, Tetrahedron, 1965, 21, 2353-2358; (c) A. Kötz and T. Steche, About the gradual oxidation of citronellol and geraniol, J. Prakt. Chem., 1924, 107, 193-210.

10 (a) T. J. Donohoe and S. Butterworth, A general oxidative cyclisation of 1,5-dienes using catalytic osmium tetroxide, Angew. Chem., Int. Ed., 2003, 42, 948-951; (b) T. J. Donohoe, J. J. G. Winter, M. Helliwell and G. Stemp, Hydrogen bonding control in the oxidative cyclisation of 1,5-dienes, Tetrahedron Lett., 2001, 42, 971-974; (c) M. de Champdore, M. Lasalvia and V. Piccialli, $\mathrm{OsO}_{4}$-Catalysed oxidative cyclisation of geranyl and neryl acetate to cis-2,5bis(hydroxymethyl)tetrahydrofurans, Tetrahedron Lett., 1998, 39, 9781-9784.

11 For cis and trans THF-diol mixture products from $\mathrm{Ru}-$ catalysed oxidative cyclisations of 1,5-dienes see: (a) V. Piccialli, T. Caserta, L. Caruso, L. Gomez-Paloma and G. Bifulco, $\mathrm{RuO}_{4}$-mediated oxidative polycyclisation of linear polyenes, a new approach to the synthesis of the bis- 
THF diol core of antitumour cis-cis adjacent bis-THF annonaceous acetogenins, Tetrahedron, 2006, 62, 1098911007; (b) S. Roth, S. Göhler, H. Cheng and C. B. W. Stark, A highly efficient procedure for ruthenium tetroxide catalysed oxidative cyclisations of 1,5-dienes, Eur. J. Org. Chem., 2005, 4109-4118; (c) S. Roth, S. Göhler, H. Cheng and C. B. W. Stark, A Highly Efficient Procedure for Ruthenium Tetroxide Catalysed Oxidative Cyclisations of 1,5-Dienes, Eur. J. Org. Chem., 2005, 4109-4118; (d) V. Piccialli and T. Caserta, Perruthenate ion. Another metal oxo species able to promote the oxidative cyclisation of 1,5dienes to 2,5-disubstituted cis-tetrahydrofurans, Tetrahedron Lett., 2004, 45, 303-308; (e) V. Piccialli and $\mathrm{N}$. Cavallo, Improved $\mathrm{RuO}_{4}$-catalysed oxidative cyclisation of geraniol-type 1,5-dienes to cis-2,5-bis(hydroxymethyl) tetrahydrofuranyldiols, Tetrahedron Lett., 2001, 42, 46954699; $(f)$ L. Albarella, D. Musumeci and D. Sica, Reactions of 1,5-dienes with ruthenium tetraoxide: stereoselective synthesis of tetrahydrofurandiols, Eur. J. Org. Chem., 2001, 997-1003; (g) P. H. J. Carlsen, T. Katsuki, V. S. Martin and K. B. Sharpless, A greatly improved procedure for ruthenium tetraoxide catalysed oxidations of organic compounds, J. Org. Chem., 1981, 46, 3936-3938.

12 Significantly, it was proposed that $\mathrm{Mn}(\mathrm{vI})$ or $\mathrm{Mn}(\mathrm{v})$ glycolate intermediate, and based on DFT calculations shown in ref. 13, enforces the 2,5-cis-relationship across the incipient THF ring during cyclisation step as indicated in Baldwin's proposed mechanism for the oxidative cyclisation of 1,5dienes.

13 A. Poethig and T. Strassner, The mechanism of the permanganate-promoted oxidative cyclisation of 1,5-dienes - A DFT study, Collect. Czech. Chem. Commun., 2007, 72, 715-727.

14 For $\mathrm{RuO}_{4}$ catalysed oxidative polycyclisation of isoprenoid polyenes for the synthesis of adjacently linked poly-THF rings see: (a) G. Bifulco, T. Caserta, L. Gomez-Paloma and V. Piccialli, $\mathrm{RuO}_{4}$-promoted syn-oxidative polycyclisation of isoprenoid polyenes: a new stereoselective cascade process, Tetrahedron Lett., 2002, 43, 9265-9269; (b) G. Bifulco, T. Caserta, L. Gomez-Paloma and V. Piccialli, Corrigendum to " $\mathrm{RuO}_{4}$-promoted syn-oxidative polycyclisation of isoprenoid polyenes: a new stereoselective cascade process", Tetrahedron Lett., 2002, 43, 9265-9269; Tetrahedron Lett., 2003, 44, 3429; (c) G. Bifulco, T. Caserta, L. Gomez-Paloma and V. Piccialli, $\mathrm{RuO}_{4}$-promoted oxidative polycyclisation of isoprenoid polyenes. A further insight into the stereochemistry of the process, Tetrahedron Lett., 2003, 44, 5499-5503; (d) T. Caserta, V. Piccialli, L. Gomez-Paloma and G. Bifulco, $\mathrm{RuO}_{4}^{-}$ catalysed oxidative polycyclisation of squalene. Determination of the configuration of the pentatetrahydrofuranyl diol product, Tetrahedron, 2005, 61, 927939; (e) V. Piccialli, N. Borbone and G. Oliviero, $\mathrm{RuO}_{4}$ catalysed oxidative polycyclisation of the Cs-symmetric isoprenoid polyene digeranyl. An unexpected stereochemical outcome, Tetrahedron, 2008, 64, 1118511192.
15 (a) A. A. Hussein, M. J. S. Phipps, C.-K. Skylaris and R. C. D. Brown, Mechanism of Os-Catalysed Oxidative Cyclisation of 1,5-Dienes, J. Org. Chem., 2019, 84, 1517315183; (b) A. Poethig and T. Strassner, Stereoselective $\mathrm{OsO}_{4}$-catalysed oxidative cyclisation of 1,5-dienes, $\mathrm{J}$. Org. Chem., 2010, 75, 1967-1973; (c) P. J. di Dio, S. Zahn, C. B. W. Stark and B. Kirchner, Understanding Selectivities in Ligand-free Oxidative Cyclisations of 1,5-and 1,6-Dienes with $\mathrm{RuO}_{4}$ from Density Functional Theory, Z. Naturforsch., B: J. Chem. Sci., 2010, 65, 367-375.

16 (a) J. Frunzke, C. Loschen and G. Frenking, Why Are Olefins Oxidized by $\mathrm{RuO}_{4}$ under Cleavage of the Carbon-Carbon Bond whereas Oxidation by $\mathrm{OsO}_{4}$ Yields cis-Diols?, J. Am. Chem. Soc., 2004, 126, 3642-3652; (b) T. Strassner and M. Drees, Rutheniumtetraoxide oxidation of alkenes a density functional theory study, J. Mol. Struct.: THEOCHEM, 2004, 671, 197-204; (c) P. O. Norrby, H. C. Kolb and K. B. Sharpless, Calculations on the reaction of ruthenium tetroxide with olefins using density functional theory (DFT). Implications for the possibility of intermediates in osmium-catalysed asymmetric dihydroxylation, Organometallics, 1994, 13, 344-347.

17 For suitability of the DFT function M06 with Ru-containing system see: (a) S. Chen, Y. Zheng, T. Cui, E. Meggers and K. N. Houk, Arylketone $\pi$-Conjugation Controls Enantioselectivity in Asymmetric Alkynylations Catalysed by Centrochiral Ruthenium Complexes, J. Am. Chem. Soc., 2018, 140, 5146-5152; (b) J. S. Cannon, L. Zou, P. Liu, Y. Lan, D. J. O'Leary, K. N. Houk and R. H. Grubbs, Carboxylate-Assisted $\mathrm{C}\left(\mathrm{sp}^{3}\right)-\mathrm{H}$ Activation in Olefin Metathesis-Relevant Ruthenium Complexes, J. Am. Chem. Soc., 2014, 136, 6733-6743; (c) Y.-F. Yang, L. W. Chung, X. Zhang, K. N. Houk and Y.-D. Wu, Ligand-Controlled Reactivity, Selectivity, and Mechanism of Cationic Ruthenium-Catalysed Hydrosilylations of Alkynes, Ketones, and Nitriles: A Theoretical Study, J. Org. Chem., 2014, 79, 8856-8864; (d) H. Miyazaki, M. B. Herbert, P. Liu, X. Dong, X. Xu, B. K. Keitz, T. Ung, G. Mkrtumyan, K. N. Houk and R. H. Grubbs, Z-Selective Ethenolysis with a Ruthenium Metathesis Catalyst: Experiment and Theory, J. Am. Chem. Soc., 2013, 135, 5848-5858.

18 Calculations on high spin multiplicity triplet and quintet were considered and shown in ESI. $\dagger$

19 (a) The ruthenate ester $\mathrm{Ru}(\mathrm{VI})$ was not isolated yet and there is no clear evidence of its formation although Sica and et al. had proposed the existence of $\mathrm{Ru}(\mathrm{vI})$ bisglycolate due to reoxidation $\mathrm{Ru}(\mathrm{vI}) \rightarrow \mathrm{Ru}(\mathrm{vIII})$ glycolate after formation of $\mathrm{Ru}(\mathrm{vI})$ glycolate (see ref. 20); (b) Like osmylation reaction that gives osmate(vi) ester, the reaction of $\mathrm{RuO}_{4}$ with double bond is called ruthenylation because it gives ruthenate(vi) ester.

20 (a) L. Albarella, F. Giordano, M. Lasalvia, V. Piccialli and D. Sica, Evidence for the existence of a cyclic ruthenium (VI) diester as an intermediate in the oxidative scission of (-)- $\alpha$-pinene with $\mathrm{RuO}_{4}$, Tetrahedron Lett., 1995, 36, 52675270; (b) V. Piccialli, D. Sica and D. Smaldone, Reaction of 7-dehydrocholesteryl acetate with $\mathrm{RuO}_{4}$. First isolation of 
a cyclic ruthenium (VI) diester, Tetrahedron Lett., 1994, 35, 7093-7096.

21 (a) The low barrier and high exergonicity of reaction reflect the experimental reaction rate aspects which is comparingly more reactive than the other metal-oxo reagents $\mathrm{MnO}_{4}{ }^{-}$and $\mathrm{OsO}_{4}$ (see ref. 13 and $15 a, b$ ), where the typical reaction time for Ru-catalysed oxidative cyclisation is 5-15 mins; (b) The barriers calculated for FRS and SRS by Dio et al., using a model of two alkenes rather than 1,5-diene, were high to be compared to the reaction time in addition to the fact their study were in accord with the general mechanistic proposal for the oxidative cyclisation of 1,5 -dienes by $\mathrm{RuO}_{4}$ (see ref. $15 c$ ).

22 It is very desirable to mention that $\mathrm{Ru}(\mathrm{vI})$ complex $\left(\left[\left(\mathrm{Me}_{3} \mathrm{tacn}\right)-\left(\mathrm{CF}_{3} \mathrm{CO}_{2}\right) \mathrm{RuO}_{2}\right] \mathrm{ClO}_{4}\right)$ was isolated by Che et al. and used in cis-dihydroxylation of alkenes in which they also isolated the $(3+2)$ cycloadduct intermediate $\mathrm{Ru}(\mathrm{Iv})$ glycolate. (See ref. 23).

23 W.-P. Yip, W.-Y. Yu, N. Zhu and C.-M. Che, Alkene cisDihydroxylation by $\left[\left(\mathrm{Me}_{3} \mathrm{tacn}\right)\left(\mathrm{CF}_{3} \mathrm{CO}_{2}\right) \mathrm{RuVIO}_{2}\right] \mathrm{ClO}_{4}$ $\left(\mathrm{Me}_{3} \mathrm{tacn}=\right.$ 1,4,7-Trimethyl-1,4,7-triazacyclononane): Structural Characterization of $(3+2)$ Cycloadducts and Kinetic Studies, J. Am. Chem. Soc., 2005, 127, 14239-14249.

24 The energy barrier for the inner sphere oxidation of $\mathrm{R}$ (VIII) glycolate $7_{[\mathbf{o x}]}$ formation from $7_{\mathrm{NaIO}}$ via $\mathrm{TS} \mathbf{1 0}$ is $39.5 \mathrm{kcal} \mathrm{mol}^{-1}$. If it is considered as an outer sphere pathway the barrier will be $33.2 \mathrm{kcal} \mathrm{mol}^{-1}$. From both manners, formation of $\mathrm{Ru}(\mathrm{vIII})$ glycolate is disfavoured.

25 A. L. Tenderholt, K. O. Hodgson, B. Hedman, R. H. Holm and E. I. Solomon, Substrate and Metal Control of Barrier Heights for Oxo Transfer to Mo and W Bis-dithiolene Sites, Inorg. Chem., 2012, 51, 3436-3442.

26 (a) T. Ishizuka, H. Kotani and T. Kojima, Characteristics and reactivity of ruthenium-oxo complexes, Dalton Trans., 2016, 45, 16727-16750; (b) W. H. Leung and C. M. Che, Highvalent ruthenium(IV) and -(VI) oxo complexes of octaethylporphyrin. Synthesis, spectroscopy, and reactivities, J. Am. Chem. Soc., 1989, 111, 8812-8818.

27 (a) B. Plietker, M. Niggemann and A. Pollrich, The acid accelerated ruthenium-catalysed dihydroxylation. Scope and limitations, Org. Biomol. Chem., 2004, 2, 1116-1124; (b) B. Plietker and $\mathrm{M}$. Niggemann, The $\mathrm{RuO}_{4}$-catalysed dihydroxylation, ketohydroxylation and mono oxidationnovel oxidation reactions for the synthesis of diols and [small alpha]-hydroxy ketones, Org. Biomol. Chem., 2004, 2, 2403-2407; (c) B. Plietker and M. Niggemann, An Improved Protocol for the $\mathrm{RuO}_{4}$-Catalysed Dihydroxylation of Olefins, Org. Lett., 2003, 5, 3353-3356.

28 Any intermolecular $(3+2)$ cycloaddition between $\mathrm{Ru}(\mathrm{VIII}) 7_{\text {[ox] }}$ and another alkene ( $E$-but-2-ene was used as a model to reduce the computational cost) to get $\mathrm{Ru}(\mathrm{vI})$ bisglycolate as called "second cycle" type was not found. However, in the Os-catalysed dihydroxylation of alkenes or oxidative cyclisation of 1,5-dienes, the second cycle pathways were noticed (see ref. $15 a$ and 29).

29 (a) P. Dupau, R. Epple, A. A. Thomas, V. V. Fokin and K. B. Sharpless, Osmium-Catalysed Dihydroxylation of
Olefins in Acidic Media: Old Process, New Tricks, Adv. Synth. Catal., 2002, 344, 421-433; (b) H. C. Kolb, M. S. VanNieuwenhze and K. B. Sharpless, Catalytic Asymmetric Dihydroxylation, Chem. Rev., 1994, 94, 24832547; (c) J. S. M. Wai, I. Marko, J. S. Svendsen, M. G. Finn, E. N. Jacobsen and K. B. Sharpless, A mechanistic insight leads to a greatly improved osmium-catalysed asymmetric dihydroxylation process, J. Am. Chem. Soc., 1989, 111, 1123-1125.

30 The $\mathrm{Ru}(\mathrm{VI})$ or $\mathrm{Ru}(\mathrm{VIII})$ glycolate have yet to be characterized or detected by any means due to the high instability. However, there is a week evidence on the existence of $\mathrm{Ru}(\mathrm{vI})$ bisglycolate. Sica et al. suggested the existence of $\mathrm{Ru}(\mathrm{vI})$ bisglycolate as an intermediate in the oxidative scission of $(-)$ - $\alpha$-pinene with $\mathrm{RuO}_{4}$ performed in acetone-water $(2: 1)$ which gives $\alpha$-ketol as the sole oxidation product (see ref. 20). Also, they proposed the same intermediate for reaction of 7-dehydrocholesteryl acetate with $\mathrm{RuO}_{4}$ to give consequently 1,2-diol and $\alpha$-ketol as final oxidation products. Their evidence was based on fast experiments monitored by NMR in which they compared their ${ }^{1} \mathrm{H}-\mathrm{NMR}$ peaks with isolated Os(vi) bisglycolate.

31 For geometry of $\mathrm{Ru}(\mathrm{vI})$ complexes see: W. P. Griffith, The Chemistry of Ruthenium Oxidation Complexes, in Ruthenium Oxidation Complexes. Catalysis by Metal Complexes, Springer Netherlands, Dordrecht, 2011, vol. 34, pp. 1-134.

32 It was not possible to locate a TS for an intermolecular ruthenylation between $\mathbf{9}_{[\mathbf{o x}][\mathbf{o x}]}$, like $7_{[\mathbf{o x}]}$ glycolate, with $E$ but-2-ene whereas this is unlike to Os-catalysed oxidative cyclisation of 1,5 -dienes.

33 In comparison to $\mathrm{Ru}(\mathrm{vI})$ glycolate 7 , the $\mathrm{Ru}(\mathrm{vI})$ glycolate 7 did not undergo intermolecular ruthenylation and this is believed due to the geometrical difference, especially angle in $\mathrm{O}=\mathrm{Ru}=\mathrm{O}$, between 7 and $\mathbf{9}_{[\mathbf{o x}]}$. The angle of $\mathrm{O}=\mathrm{Ru}=\mathrm{O}$ in $\mathrm{Ru}(\mathrm{vI})$ diolate $\mathbf{9}_{[\mathbf{o x}]}$ is $119.4^{\circ}$ and $\mathrm{Ru}(\mathrm{vI})$ glycolate 7 is $125.7^{\circ}$. 34 Due to the difficulty in locating the TS 6a using the DFT functional M06 (M06/cc-pVDZ/LANL2DZ), both TSs 6a and d-6a have been optimised in gas phase with M06-2X (M062X/cc-pVDZ/LANL2DZ) followed by single point energy calculations in THF with the M06 functional ((SMD)-M06// aug-cc-pVDZ/LANL2DZ).

35 M. J. Frisch, G. W. Trucks, H. B. Schlegel, G. E. Scuseria, M. A. Robb, J. R. Cheeseman, G. Scalmani, V. Barone, B. Mennucci, G. A. Petersson, H. Nakatsuji, M. Caricato, X. Li, H. P. Hratchian, A. F. Izmaylov, J. Bloino, G. Zheng, J. L. Sonnenberg, M. Hada, M. Ehara, K. Toyota, R. Fukuda, J. Hasegawa, M. Ishida, T. Nakajima, Y. Honda, O. Kitao, H. Nakai, T. Vreven, J. A. Montgomery Jr, J. E. Peralta, F. Ogliaro, M. J. Bearpark, J. Heyd, E. N. Brothers, K. N. Kudin, V. N. Staroverov, R. Kobayashi, J. Normand, K. Raghavachari, A. P. Rendell, J. C. Burant, S. S. Iyengar, J. Tomasi, M. Cossi, N. Rega, N. J. Millam, M. Klene, J. E. Knox, J. B. Cross, V. Bakken, C. Adamo, J. Jaramillo, R. Gomperts, R. E. Stratmann, O. Yazyev, A. J. Austin, R. Cammi, C. Pomelli, J. W. Ochterski, R. L. Martin, K. Morokuma, V. G. Zakrzewski, G. A. Voth, 
P. Salvador, J. J. Dannenberg, S. Dapprich, A. D. Daniels, Ö. Farkas, J. B. Foresman, J. V. Ortiz, J. Cioslowski and D. J. Fox, Gaussian 09 Revision D.01, Gaussian, Inc., Wallingford, CT, USA, 2013.

36 (a) Y. Zhao and D. G. Truhlar, Applications and validations of the Minnesota density functionals, Chem. Phys. Lett., 2011, 502, 1-13; (b) Y. Zhao and D. G. Truhlar, The M06 suite of density functionals for main group thermochemistry, thermochemical kinetics, noncovalent interactions, excited states, and transition elements: two new functionals and systematic testing of four M06-class functionals and 12 other functionals, Theor. Chem. Acc., 2007, 120, 215-241.

37 (a) T. H. Dunning, Gaussian basis sets for use in correlated molecular calculations. I. The atoms boron through neon and hydrogen, J. Chem. Phys., 1989, 90, 1007-1023; (b) R. A. Kendall, T. H. Dunning and R. J. Harrison, Electron affinities of the first-row atoms revisited. Systematic basis sets and wave functions, J. Chem. Phys., 1992, 96, 67966806; (c) D. E. Woon and T. H. Dunning, Gaussian basis sets for use in correlated molecular calculations. III. The atoms aluminum through argon, J. Chem. Phys., 1993, 98, 1358-1371.

38 (a) P. J. Hay and W. R. Wadt, Ab initio effective core potentials for molecular calculations. Potentials for the transition metal atoms Sc to $\mathrm{Hg}$, J. Chem. Phys., 1985, 82, 270-283; (b) W. R. Wadt and P. J. Hay, Ab initio effective core potentials for molecular calculations, Potentials for main group elements $\mathrm{Na}$ to $\mathrm{Bi}, \mathrm{J}$. Chem. Phys., 1985, 82, 284-298; (c) P. J. Hay and W. R. Wadt, Ab initio effective core potentials for molecular calculations. Potentials for $\mathrm{K}$ to Au including the outermost core orbitals, J. Chem. Phys., 1985, 82, 299-310.

39 (a) H. B. Schlegel, Optimisation of equilibrium geometries and transition structures, J. Comput. Chem., 1982, 3, 214218; (b) X. Li and M. J. Frisch, Energy-Represented Direct Inversion in the Iterative Subspace within a Hybrid Geometry Optimisation Method, J. Chem. Theory Comput., 2006, 2, 835-839.

40 A. V. Marenich, C. J. Cramer and D. G. Truhlar, Universal Solvation Model Based on Solute Electron Density and on a Continuum Model of the Solvent Defined by the Bulk Dielectric Constant and Atomic Surface Tensions, J. Phys. Chem. B, 2009, 113, 6378-6396.

41 (a) H. P. Hratchian and H. B. Schlegel, Accurate reaction paths using a Hessian based predictor-corrector integrator, J. Chem. Phys., 2004, 120, 9918-9924; (b) M. A. Collins, Molecular potential-energy surfaces for chemical reaction dynamics, Theor. Chem. Acc., 2002, 108, 313-324; (c) K. Fukui, The path of chemical reactions - the IRC approach, Acc. Chem. Res., 1981, 14, 363-368.

42 C. Y. Legault, CYLview 1.0b, Universitéde Sherbrooke, Canada, 2009, http://www.cylview.org. 